\title{
Reconstrução atípica de laringe: avaliação funcional pós-laringectomia supratraqueal ampliada com cricoglossohioidopexia
}

\section{Atypical reconstruction of the larynx: functional assessment after enlarged supratracheal laryngectomy with cricoglossohioidopexy}

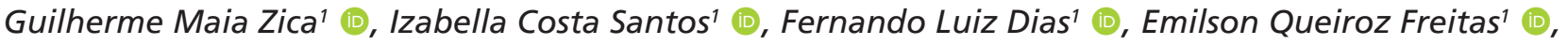 \\ Andressa Silva de Freitas ${ }^{1}$ (i)
}

\begin{abstract}
RESUMO
Em tumores transglóticos estendidos para base de língua, a indicação para ressecção do osso hioide descarta a possibilidade de realização de uma laringectomia parcial horizontal clássica, devido ao grande risco de complicações pulmonares oriundas de uma disfagia grave. O objetivo deste estudo foi descrever os aspectos funcionais de deglutição e voz de um paciente submetido à laringectomia supratraqueal ampliada com cricoglossohioidopexia. Trata-se de um homem de 69 anos, com tumoração transglótica na hemilaringe direita, submetido à laringectomia supratraqueal ampliada, com ampliação para base de língua, osso hioide e aritenoide direita. Na videofluoroscopia da deglutição, observou-se aspiração silente para líquido fino durante a deglutição e resíduo em base de língua, valécula, aritenoide, esfíncter esofágico superior e recessos piriformes em todas as consistências e volumes. Na videolaringoscopia, observou-se voz por meio da vibração da unidade cricoaritenóidea esquerda, associada à base de língua e constritores da faringe. No protocolo Consensus Auditory-Perceptual Evaluation of Voice (CAPE V), notou-se grau moderado de rouquidão e soprosidade. O paciente apresentou preservação parcial das funções laríngeas, grau moderado de disfonia e alimentação e hidratação exclusivas por via oral, com sólidos macios e líquido espessado em néctar, sem prejuízos à saúde pulmonar, até o momento.
\end{abstract}

Palavras-chave: Neoplasias de cabeça e pescoço; Laringectomia; Disfagia; Voz; Estudo de caso

\begin{abstract}
In transglottic tumors extended to the base of the tongue, the indication for resection of the hyoid bone rules out the possibility of performing a classic horizontal partial laryngectomy due to the high risk of pulmonary complications resulting from severe dysphagia. This study aims to describe the functional aspects of swallowing and voice of a patient undergoing an enlarged supratracheal laryngectomy with cricoglossohioidopexy. This is a 69-year-old man with a transglottic tumor in the right hemilarynx, who underwent an extended supratracheal laryngectomy with enlargement to the base of the tongue, hyoid bone and right arytenoid. In swallowing videofluoroscopy, silent aspiration was observed in fine liquid and residue on the basis of tongue, valecule, arytenoid, upper esophageal sphincter and pyriform recesses in all consistencies and volumes. In videolaryngoscopy, a voice was observed through the vibration of the left cricoarytenoid unit associated with the base of the tongue and constrictors of the pharynx. The Consensus Auditory-Perceptual Evaluation of Voice (CAPE V) showed a moderate degree of hoarseness and breathiness. The patient had partial preservation of laryngeal functions, with a moderate degree of dysphonia and exclusive oral feeding and hydration with soft solids and thickened liquid in nectar without impairing lung health until the study.
\end{abstract}

Keywords: Head and neck cancer; Laryngectomy; Dysphagia; Voice; Case reports

Trabalho realizado no Instituto Nacional de Câncer - INCA - Rio de Janeiro (RJ), Brasil.

${ }^{1}$ Instituto Nacional de Câncer - INCA - Ministério da Saúde - Rio de Janeiro (RJ), Brasil.

Conflito de interesses: Não.

Contribuição dos autores: GMZ concepção e desenho do trabalho, aquisição, análise e interpretação dos dados da pesquisa, redação e revisão crítica com contribuição intelectual e aprovação final da versão para publicação; ICS concepção e desenho do trabalho, revisão crítica com contribuição intelectual e aprovação final da versão para publicação; FLD redação e revisão crítica com contribuição intelectual; EQF cirurgião responsável pela execução da técnica descrita e redação e revisão crítica com contribuição intelectual; ASF participou, na condição de orientadora, da concepção e desenho do trabalho, aquisição, análise e interpretação dos dados da pesquisa, redação e revisão crítica com contribuição intelectual e aprovação final da versão para publicação.

Financiamento: Nada a declarar.

Autor correspondente: Guilherme Maia Zica. E-mail: guilhermemaiafono@gmail.com

Recebido: Outubro 28, 2020; Aceito: Janeiro 05, 2021 


\section{INTRODUÇÃO}

As diversas formas de tratamento desenvolvidas no século XX potencializaram o cuidado e aumentaram, significativamente, a sobrevida de pacientes com câncer de cabeça e pescoço ${ }^{(1,2)}$. Devido aos desfechos negativos oriundos da perda permanente da voz laríngea, disfagia e menor qualidade de vida, os protocolos de preservação de órgãos questionam a indicação de laringectomias totais ${ }^{(3)}$.

A laringectomia parcial supracricóidea (LSC) foi introduzida por Majer em $1959^{(4)}$ e aperfeiçoada ao longo dos anos, como forma de opção à laringectomia total( ${ }^{(2)}$. Com o aprimoramento da técnica e sua consolidação por meio do adequado controle oncológico e reprodutibilidade, surgiram novas tentativas de adaptações para ampliação do perfil de paciente indicado. A laringectomia parcial supratraqueal (LST) é uma intervenção cirúrgica atual e análoga à LSC como adaptação para o tratamento de tumores de laringe com extensão subglótica e com invasão na cartilagem cricoide de estágios intermediário/avançado ${ }^{(2-5)}$. A técnica cirúrgica, originalmente descrita na literatura por Laccourreye em 1994, consiste na ressecção de toda a cartilagem tireoide e espaço paraglótico, com a preservação de parte da cartilagem cricoide e do osso hioide, podendo-se preservar, ou não, a epiglote e, pelo menos, uma cartilagem aritenoide ${ }^{(5)}$. Sua reconstrução é descrita com duas variações: 1 - traqueohioidopexia (THP), em que há manutenção de ambas ou apenas uma unidade cricoaritenoide; 2 - traqueohioidoepiglotopexia (THEP), em que a ressecção prevê a preservação da epiglote, mantendo toda a unidade cricoartenóidea ou apenas uma unidade cricoartenóidea ${ }^{(5,6)}$.

As complicações funcionais mais recorrentes das laringectomias parciais abertas horizontais (LSC e LST) são, sobretudo, as que se referem às funções respiratórias e de deglutição $0^{(1,3)}$. Em casos cuja ressecção é mais ampla que a prevista pela técnica cirúrgica, como em casos já descritos de LST que retiram parte de base de língua, tecido adjacente ou aritenoide, nomeia-se o procedimento de LST extendido, alargada ou modificada ${ }^{(2,6-9)}$.

Em tumores transglóticos estendidos para base de língua, a indicação de ressecção do osso hioide descarta a possibilidade de realização de uma laringectomia parcial clássica, devido à complexidade da fixação da cartilagem cricoide e reconstrução das estruturas remanescentes. Essa neolaringe pode, teoricamente, gerar um grande risco de complicações pulmonares, devido à disfagia grave ${ }^{(7)}$. Portanto, tumores com essas características são tradicionalmente indicados para a realização de uma laringectomia total, o que contribui para graves prejuízos funcionais e de qualidade de vida.

Com o objetivo de reduzir as indicações de laringectomia total e expandir a aplicação de laringectomias parciais no tratamento do câncer de laringe, foi realizada uma ampliação da técnica para suprir a demanda funcional e psicossocial de um paciente. Portanto, o objetivo deste relato de caso foi descrever os aspectos funcionais de deglutição e voz de um paciente submetido à laringectomia supratraqueal ampliada com cricoglossohioidopexia, técnica de sucesso e inédita no mundo.

\section{APRESENTAÇÃO DO CASO CLÍNICO}

O estudo foi aprovado pelo Comitê de Ética e Pesquisa da instituição com o número 89042418.7.0000.5274. O paciente concordou em participar do estudo e assinou o Termo de Consentimento Livre e Esclarecido.

Paciente do sexo masculino, 69 anos, com ensino fundamental incompleto, sem antecedentes de câncer na família, tabagista e etilista há aproximadamente 35 anos, com histórico prévio de tumor de prega vocal direita, sendo tratado com microcirurgia a laser e radioterapia com intensidade modulada (IMRT com 66 Gy em 33 frações). Após o tratamento, o paciente manteve o hábito de tabagismo (Figura 1). Permaneceu livre de doença por, aproximadamente, dez anos.

Durante o acompanhamento médico do controle oncológico hospitalar, exames identificaram recidiva com tumoração transglótica em laringe. Pela videolaringoscopia, observou-se lesão infiltrativa em prega ariepiglótica direita, face laríngea da epiglote com invasão em seio piriforme direito, estendendo-se superiormente para base de língua (Figura 2). Observou-se, também, mobilidade preservada de ambas as pregas vocais e de aritenoide esquerda.

Durante o planejamento terapêutico, o paciente demonstrou descontentamento em realizar o tratamento preconizado laringectomia total -, por permanência da traqueostomia e perda da voz. Nesse caso, houve a sugestão de realizar uma LST alargada como uma proposta mais conservadora, tendo em vista que a re-irradiação não era uma opção viável.

Após deliberação clínica multiprofissional e esclarecimento dos riscos e benefícios, o paciente foi submetido a uma LST modificada, alargada para a base de língua em campo estreito. Foram retiradas as estruturas: cartilagem tireoide, epiglote, pregas ariepiglóticas, seios piriformes, pregas vocais e aritenoide direita. Não foi realizado esvaziamento cervical. A extensão da cirurgia culminou na ressecção de, aproximadamente, $50 \%$ da cartilagem cricoide e do osso hioide do lado direito. Com isso, as estruturas laríngeas remanescentes do lado esquerdo foram fixadas na base da língua, devido ausência ipsilateral do osso hioide. Não houve intercorrências no pós-operatório (Figura 3).

O paciente recebeu alta hospitalar após 22 dias do procedimento cirúrgico e a traqueostomia foi mantida por 75 dias. O suporte nutricional nos primeiros dois meses pós-cirurgia foi por meio

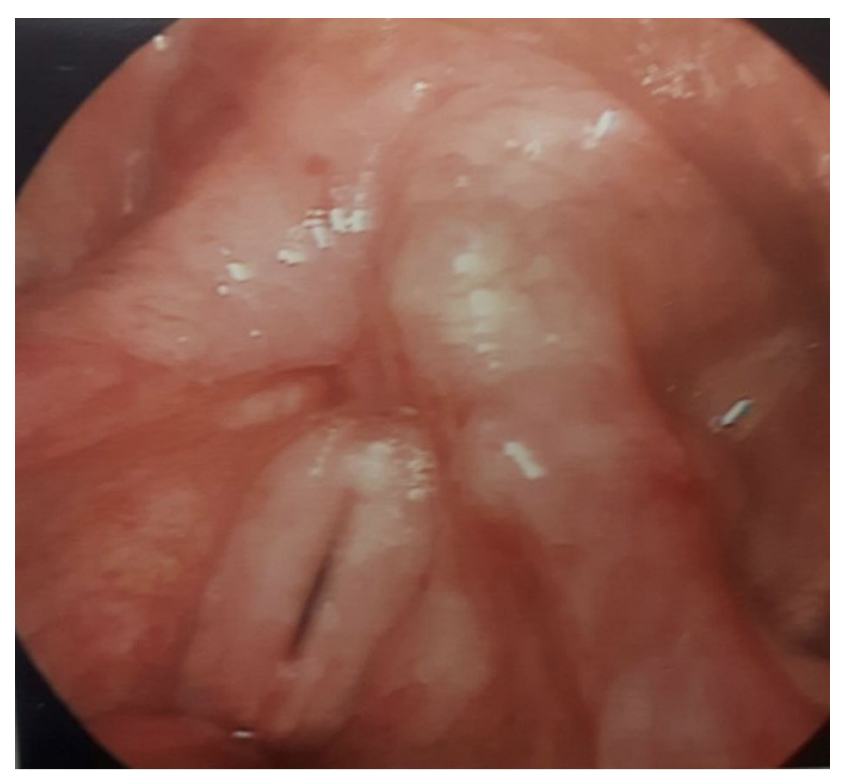

Figura 1. Videolaringoscopia de paciente em controle oncológico. Observa-se edema em região laríngea e faríngea, devido ao tabagismo 


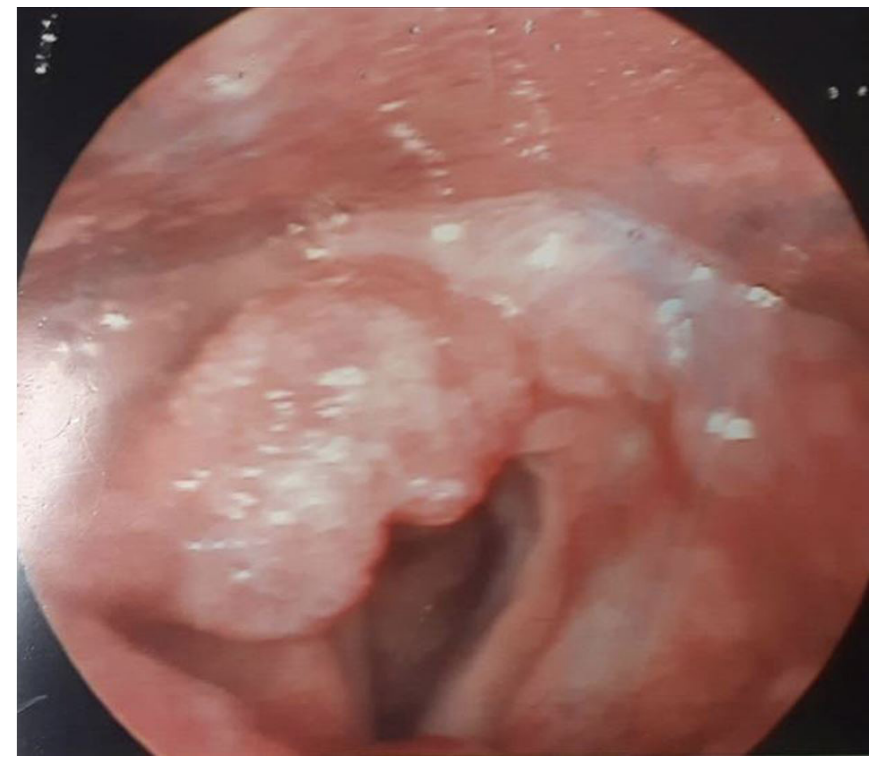

Figura 2. Videolaringoscopia no pré-operatório em controle oncológico. Visão das estruturas afetadas pela neoplasia: lesão infiltrativa em prega ariepiglótica direita, face laríngea da epiglote com invasão em seio piriforme direito, estendendo-se superiormente para base de língua

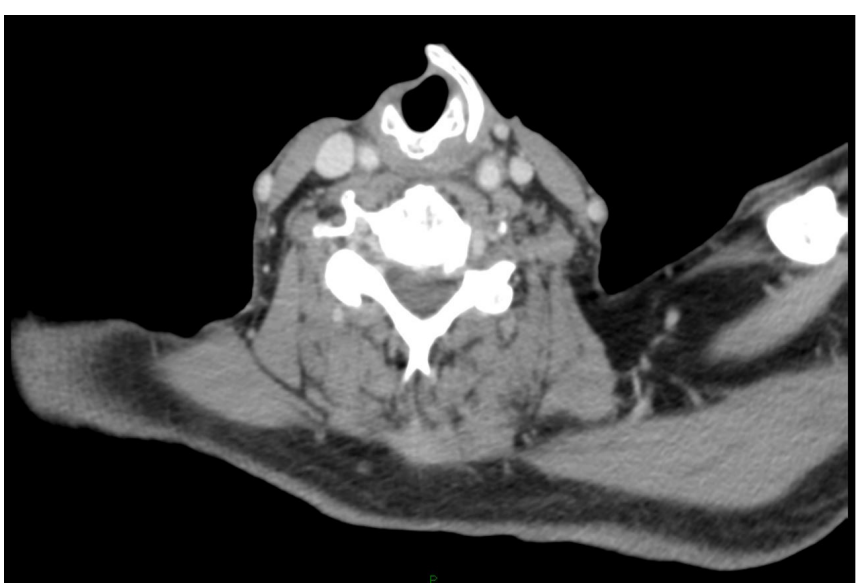

Figura 3. Tomografia computadorizada no pós-operatório: ressecção da cartilagem cricoide e osso hioide

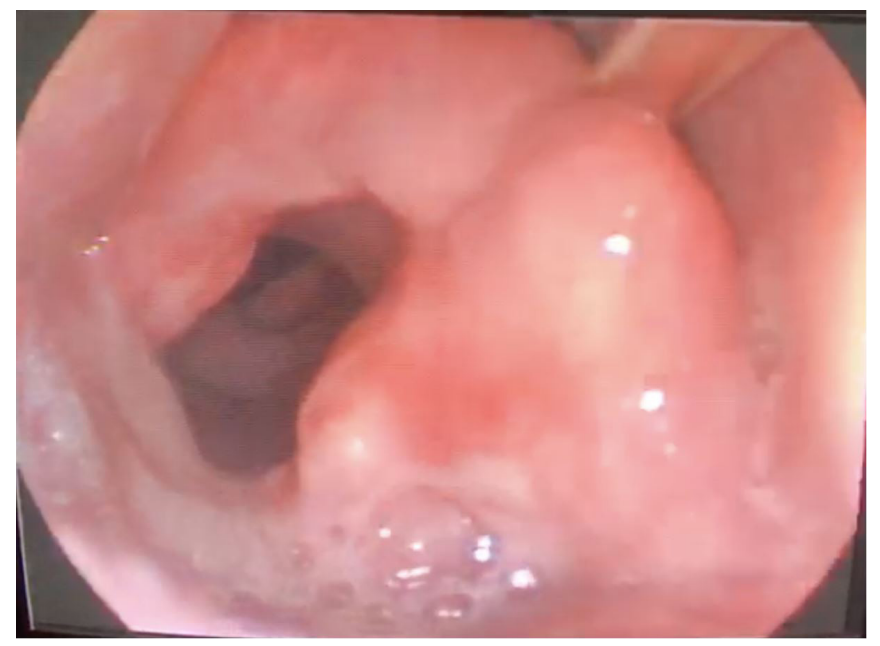

Figura 4. Videolaringoscopia no pós-operatório em controle oncológico. Observa-se a preservação de aritenoide esquerda e estase de saliva em recessos faríngeos do lado esquerdo da sonda nasogástrica (SNG) exclusiva e, após esse período, ele permaneceu três meses com SNG e alimentação via oral, na consistência pastosa liquidificada e líquido espessado em mel. A SNG apresentou permanência de 92 dias e, depois disso, o paciente seguiu com via oral exclusiva, evoluindo consistências progressivamente até a alimentação com sólidos macios e líquido espessado em néctar. $\mathrm{O}$ paciente permaneceu funcionalmente estável com o uso de espessante alimentar.

Exatamente após a cirurgia e até o momento da realização do estudo, o paciente esteve sob os cuidados da equipe de Fonoaudiologia, sendo exposto à reabilitação da deglutição e da voz, devido, principalmente, a um fechamento laríngeo incompleto, proveniente do tratamento cirúrgico.

No exame clínico do pós-operatório imediato, observouse alteração grave da mobilidade da língua, sendo tracionada posteriormente pela pexia em base de língua. Algumas estratégias tornam-se relevantes, como exercícios para mobilidade e força de língua, assim como as manobras supraglótica e supersupraglótica, que possibilitam a maior amplitude de movimento e duração do fechamento do vestíbulo laríngeo ${ }^{(1,3)}$.

Para avaliar os resultados funcionais seis meses após a cirurgia, realizou-se a videofluoroscopia da deglutição e a videolaringoscopia.

O paciente realizou o exame de videofluoroscopia da deglutição já com via oral exclusiva, após seis meses de cirurgia. Foi utilizado o Aparelho de Raios-X Telecomandado Siemens Axion Iconos MD (Número de Série 13020). Todos os segmentos de vídeo foram gravados em um plano de visualização lateral, com taxa de captura de imagem de 30 quadros (frames) por segundo. A Escala de Penetração e Aspiração desenvolvida por Rosenbek, em 1996, foi utilizada como parâmetro de segurança da deglutição ${ }^{(10)}$. Foi considerado resíduo o material acumulado em diferentes regiões da faringe após o término da deglutição ${ }^{(11)}$. $\mathrm{O}$ contraste foi oferecido em copo, utilizando-se diluições de sulfato de bário (SB) a 100\%, Bariogel ${ }^{\circledR}$, água mineral e espessante Resource ${ }^{\circledR}$ Thicken Up Clear. A avaliação foi composta pela oferta de três consistências, sendo elas: líquida, em $5 \mathrm{ml}(2,5 \mathrm{ml}$ de água $+2,5 \mathrm{ml}$ de SB), $10 \mathrm{ml}$ (5 $\mathrm{ml}$ de água $+5 \mathrm{ml}$ de SB) e $20 \mathrm{ml}(10 \mathrm{ml}$ de água $+10 \mathrm{ml} \mathrm{de} \mathrm{SB})$; semi líquido, em $5 \mathrm{ml}$ de SB, $10 \mathrm{ml}$ de $\mathrm{SB}$ e $20 \mathrm{ml}$ de SB; pastosa, em $5 \mathrm{ml}$ (5 $\mathrm{ml}$ de $\mathrm{SB}+1,2 \mathrm{~g}$ de espessante), $10 \mathrm{ml}$ (10 $\mathrm{ml}$ de $\mathrm{SB}+2,4 \mathrm{~g}$ de espessante) e $20 \mathrm{ml}(20 \mathrm{ml}$ de $\mathrm{SB}+3,6 \mathrm{~g}$ de espessante). $\mathrm{O}$ indivíduo foi posicionado sentado em visão lateral, o mais próximo possível do tampo da mesa e do intensificador, evitando-se, dessa forma, distorções da imagem fluoroscópica.

No exame objetivo da deglutição foi observada aspiração silente em líquido fino durante a deglutição e resíduo em base de língua, valécula, aritenoide, esfíncter esofágico superior e recessos piriformes em todas as consistências e volumes, principalmente com semi líquido e pastoso. Apesar dos resultados funcionais descritos, o paciente referiu queixas relacionadas à deglutição apenas com líquido fino.

Quanto à videolaringoscopia, representada na Figura 4, observou-se a preservação da voz a partir da vibração da unidade cricoaritenóidea esquerda, associada às estruturas remanescentes (base de língua e constritores da faringe). Na análise perceptivoauditiva, por meio da Consensus Auditory-Perceptual Evaluation of Voice (CAPE V) ${ }^{(6)}$, notou-se qualidade vocal rouca e soprosa. O paciente apresentou boa inteligibilidade de fala e desvio de pitch e loudness (voz grave e intensidade reduzida). 
Até o momento da escrita deste relato, o paciente mantinha-se em controle ambulatorial, sem câncer há dois anos e ausência de complicações pulmonares.

\section{DISCUSSÃO}

Os desfechos funcionais e oncológicos da LSC e LST são favoráveis em tumores supraglótico e glótico ${ }^{(3-6,12)}$. Tumores transglóticos que acometem amplamente o espaço pré-epiglótico, o osso hioide, imobilizam, pelo menos, uma articulação cricoaritenóidea, acometem a cartilagem tireoide e invadem o espaço subglótico, envolvendo a cartilagem cricoide, geralmente requerem uma laringectomia total ${ }^{(7)}$.

É possível compreender que um desfecho desfavorável e deficit na funcionalidade da laringe ainda caracterizam um prognóstico restrito dos pacientes com a doença em seu estágio avançado, devido à perda ou alteração da voz e presença de quadros de disfagia que comprometem, de forma significativa, a qualidade de vida ${ }^{(1,6)}$.

A fim de solucionar esses prejuízos, a técnica de LST ampliada tem sido utilizada como alternativa às laringectomias totais $^{(6,7)}$. No entanto, a invasão da base de língua e osso hioide mantém a contraindicação, devido a fatores como dificuldades/ complicações de reconstrução da neolaringe e aspiração maciça intratável ${ }^{(7)}$.

A suspensão anteroposterior da neolaringe é uma medida protetiva das vias aéreas inferiores e, portanto, sua fixação na base da língua não garante de forma segura esse posicionamento, sendo necessária a fixação em uma base óssea ${ }^{(4,5,7)}$. A metodologia descrita neste estudo foi adaptada mediante a descrição de Laccourreye de $1994^{(5)}$. A cartilagem cricoide foi aproximada na porção do osso hioide remanescente do lado esquerdo e fixada na base da língua do lado direito, na tentativa de assegurar a elevação e anteriorização do remanescente laríngeo.

Técnica semelhante foi abordada na literatura por Hafız e colaboradores ${ }^{(7)}$, com uma reconstrução nomeada de cricoglossomandibulopexia. Neste estudo, realizou-se a ressecção completa do osso hioide e a fixação da neolaringe na mandíbula (base óssea) e na base de língua. Entretanto, as estruturas provenientes da pexia foram rompidas após a cirurgia.

No câncer de laringe, a cirurgia deve comprovar resultados funcionais aceitáveis em termos de voz e deglutição, para manter e justificar uma cirurgia parcial ${ }^{(1,3,6)}$. Desse modo, uma reconstrução que englobe a ressecção do anel cricoide e do osso hioide na LST em tumores transglóticos minimamente invasivos na subglote é, porventura, um desafio para a equipe de reabilitação ${ }^{(1,6,7)}$

Existem fatores críticos na recuperação da deglutição após as laringectomias parciais: o fechamento da via aérea na entrada da neolarínge (isto é, o espaço entre a cartilagem aritenoide e a base de língua) e o contato da base de língua com a parede posterior da faringe ${ }^{(1,6)}$. Devido às alterações anatômicas oriundas da reconstrução, a ação esfincteriana é proporcionada pela aproximação da cartilagem aritenoide móvel (girando para frente e para dentro), da epiglote (inclinando para trás) e das estruturas remanescentes ${ }^{(2,3)}$.

A literatura que trata das laringectomias parciais afirma que pacientes com deglutição funcional apresentaram tempo de trânsito faríngeo total mais longo, abertura lateral mais estreita do esfíncter esofágico superior e um contato mais fraco entre a base de língua e a parede posterior de faringe. As pressões faríngeas dependem da ação da válvula velofaríngea, da protrusão da base de língua e da contração dos constritores da faringe. Portanto, pode-se especular que a retração incompleta da base de língua resultou em menor pressão faríngea, prolongando a duração do tempo total de trânsito, reduzindo a abertura lateral do esfíncter esofágico superior e a elevação do osso hioide ${ }^{(3,6)}$. Essa nova fisiologia provavelmente proporcionou a grande quantidade de resíduos pós-deglutição no indivíduo avaliado.

As complicações funcionais mais frequentes na LST estão, geralmente, relacionadas à respiração, voz e deglutição. Assim, a presença de resíduo e aspiração na análise de deglutição do paciente do presente estudo já era parcialmente prevista, conforme relatado por alguns autores ${ }^{(6)}$. Há relatos na literatura de que, aproximadamente, $40 \%$ dos pacientes submetidos à LSC e LST apresentam aspiração crônica, principalmente com líquidos finos, achado que se justifica pelo fechamento incompleto da laringe, devido à retirada total das pregas vocais e vestibulares ${ }^{(6,7,12)}$.

A voz dos pacientes submetidos à LST é produzida por meio da passagem do ar pela neolaringe durante a expiração, promovendo a vibração da mucosa e estruturas remanescentes, como as aritenoides e a faringe ${ }^{(2,6)}$. É consenso na literatura a presença de algum grau de rugosidade, rouquidão e soprosidade na emissão da voz desses pacientes, devido à retirada de, aproximadamente, $70 \%$ da laringe ${ }^{(6)}$. Portanto, observa-se a manutenção de uma voz funcional e laríngea, considerada como sucesso na LST.

É importante ressaltar que a presença de aspiração e resíduo não afetaram as condições gerais de saúde do paciente, até o momento da avaliação. O sucesso terapêutico (nutrição e hidratação exclusivas por via oral e voz laríngea funcional) foi possível por meio do atendimento fonoaudiológico constante em todos os momentos do tratamento e medidas para compensar os danos funcionais. Tais estratégias terapêuticas precisam superar os prejuízos da aspiração e orientações, como manter uma vida ativa, alimentação saudável, higiene oral rigorosa e acompanhamento médico, fonoaudiológico, fisioterápico e nutricional são essenciais.

A decisão da realização da LST ampliada, neste caso, baseou-se, principalmente, na intenção de preservar as funções laríngeas, devido à demanda do paciente, sendo que observou-se mobilidade de ambas as pregas vocais e de aritenoide esquerda em exame de videolaringoscopia pré-cirurgia. Para tumores transglóticos com invasão da base de língua, hioide e espaço pré-epiglótico, a LST estentida com reconstrução por meio da cricoglossohioidopexia apresentou repercussões oncológicas e funcionais favoráveis no caso descrito.

O paciente se manteve em controle oncológico e com acompanhamento multiprofissional para manutenção funcional até o momento da realização deste estudo.

\section{COMENTÁRIOS FINAIS}

Os desfechos funcionais e oncológicos da laringectomia supratraqueal com reconstrução por meio da cricoglossohioidopexia foram favoráveis para um tumor transglótico com invasão em base de língua. O paciente apresentou preservação parcial das funções laríngeas, grau moderado de disfonia e disfagia e alimentação e hidratação exclusivas por via oral. A equipe multiprofissional especializada, incluindo a Fonoaudiologia, é essencial, devido ao complexo processo de reabilitação. 


\section{REFERÊNCIAS}

1. Zica GM, Freitas AS. Deliberações clínicas na atuação em pacientes com câncer de cabeça e pescoço: atualidades e desafios na disfagia. Distúrb Comun. 2019;31(4):693-7. http://dx.doi.org/10.23925/21762724.2019v31i4p693-697.

2. Zica GM, De Freitas AS, Lopes WFM, Da Silva BLM, Souza FGR, Freitas EQ, et al. Aspectos funcionais e epidemiológicos da deglutição na laringectomia supratraqueal extendida com traqueohiodoepiglotopexia. Distúrb Comun. 2019;31(1):87-94. http://dx.doi.org/10.23925/21762724.2019v31i1p87-94.

3. Freitas AS, Zica GM. Eficiência da deglutição na laringectomia parcial horizontal: pensando além da segurança. Distúrb Comun. 2019;31(3):52931. http://dx.doi.org/10.23925/2176-2724.2019v31i3p529-531.

4. Majer H. Technique de laryngectomie permettant de conserver la permeabilite' respiratoire la cricohyoido-pexie. Ann Otolaryngol. 1959 Jul-Ago;76:677-81. PMid:14420078.

5. Laccourreye O, Ross J, Brasnu D, Chabardes E, Kelly JH, Laccourreye H. Extended supracricoid partial laryngectomy with tracheocricohyoidoepiglottopexy. Acta Otolaryngol. 1994;114(6):66974. http://dx.doi.org/10.3109/00016489409126124. PMid:7879627.

6. Zica GM, Freitas AS, Silva ACAE, Dias FL, Santos IC, Freitas EQ, et al. Swallowing, voice and quality of life of patients submitted to extended supratracheal laryngectomy. Einstein (Sao Paulo). 2020;18:eAO5390. http://dx.doi.org/10.31744/einstein journal/2020AO5390. PMid:32428067.

7. Hafiz G, Başaran B, Ulusan M, Çomoğlu Ş. A new extended supracricoid laryngectomy technique for tongue base and hyoid bone involvement: crico-glosso-mandibulopexy technique. Kulak Burun Bogaz Ihtis Derg. 2014;24(3):181-4. http://dx.doi.org/10.5606/kbbihtisas.2014.64614. PMid:25010810.

8. Rifai M. Extended supracricoid laryngectomy with excision of both arytenoids: the modified reconstructive laryngectomy. Acta Otolaryngol 2007;127(6):642-50. http://dx.doi.org/10.1080/00016480601001940. PMid:17503235.

9. Zhao X, Ji W. The application of extended supraglottic horizontal partial laryngectomy in advanced laryngeal carcinoma and vallecula carcinoma. Lin Chung Er Bi Yan Hou Tou Jing Wai Ke Za Zhi. 2015;29(7):593-6. PMID: 26201180.

10. Rosenbek JC, Robbins JA, Roecker EB, Coyle JL, Wood JL. A penetration-aspiration scale. Dysphagia. 1996;11(2):93-8. http:// dx.doi.org/10.1007/BF00417897. PMid:8721066.

11. Logemann JA. The evaluation and treatment of swallowing disorders. Curr Opin Otolaryngol Head Neck Surg. 1998;6(6):395-400. http:// dx.doi.org/10.1097/00020840-199812000-00008.

12. Bagwell K, Leder SB, Sasaki CT. Is partial laryngectomy safe forever? Am J Otolaryngol. 2015;36(3):437-41. http://dx.doi.org/10.1016/j. amjoto.2014.11.005. PMid:25595047. 